\title{
Deaths of colon neuroendocrine tumors are associated with increasing metastatic lymph nodes and lymph node ratio
}

\author{
Changchun Xiao ${ }^{1 \#}$, Baorong Song ${ }^{2,3,4 \#}$, Peipei $\mathrm{Yi}^{5 \#}$, Yangyang $\mathrm{Xie}^{2,3,4}$, Biqing $\mathrm{Li}^{6}$, Peng Lian ${ }^{7}$, \\ Shaoqing Ding ${ }^{2,3,4}$, Yuanming Lu $^{5}$
}

${ }^{1}$ Department of General Surgery, Shanghai Electric Power Hospital, Shanghai, China; ${ }^{2}$ Department of Gastroenterology, HwaMei Hospital, University of Chinese Academy of Sciences, Ningbo, China; ${ }^{3}$ Ningbo Institute of Life and Health Industry, University of Chinese Academy of Sciences, Ningbo, China; ${ }^{4}$ Key Laboratory of Diagnosis and Treatment of Digestive System Tumors of Zhejiang Province, Ningbo, China; ${ }^{5}$ Department of Toxicology, School of Public Health, Guilin Medical University, Guilin, China; ${ }^{6}$ Key Laboratory of Systems Biology, Shanghai Institutes for Biological Sciences, Chinese Academy of Sciences, Shanghai, China; ${ }^{7}$ Department of Colorectal Surgery, Fudan University Shanghai Cancer Center, Shanghai, China

Contributions: (I) Conception and design: C Xiao, B Song, Y Lu; (II) Administrative support: Y Lu; (III) Provision of study materials or patients: C Xiao, B Song, P Yi, (IV) Collection and assembly of data: Y Xie, P Lian, S Ding; (V) Data analysis and interpretation: C Xiao, B Song, B Li, P Lian; (VI) Manuscript writing: All authors; (VII) Final approval of manuscript: All authors.

\#These authors contributed equally to this work.

Correspondence to: Yuanming Lu, PhD. Department of Toxicology, School of Public Health, Guilin Medical University, Guilin, China. Email: bobojpn2002@163.com.

Background: Colon neuroendocrine tumors (NETs) are uncommon. Currently, the impact of the number of metastatic lymph nodes (LNs) and lymph node ratio (LNR) on survival has been well investigated in other colon malignancies, but both remain nebulous for patients with colon NETs.

Methods: Surgically resected patients with histologically proven nonmetastatic colon NETs were queried from the Surveillance, Epidemiology, and End Results database between 1988 and 2011. Patients with lymph nodes involved were investigated and categorized into four LNs-based classifications $(\leq 4,>4-10,>10-13$, and $>13)$ or three LNR-based subgroups $(\leq 0.51,>0.51-0.71$, and $>0.71)$ according to the threshold, determined by Harrell's C statistic. Univariate and multivariate survival analyses were performed by log-rank test and Cox stepwise regression analysis, respectively.

Results: Eight hundred fifty-one patients met the inclusion criteria. Among them, higher LNR and LNs classification are associated with a worse prognosis. The 10-year NETs-specific survival rate was $78.3 \%$ (74.2-82.6\%), 61.3\% (52.4-71.7\%), 40.8\% (20.7-80.7\%) for patients in the $\leq 4,>4-10$, and $10-13$ LNs groups, respectively. When patients were classified with LNR, the observed 10-year NETs-specific survival rate was $79.9 \%(74.8-85.5 \%)$ for $\leq 0.51,57.4 \%$ (43.8-75.2\%) for $>0.51-0.71$, and $40.0 \%(31.0-51.5 \%)$ for $>0.71$. In stratified analysis, higher LNs and LNR groups have worse prognosis only in patients with advanced T stage (T3-T4). Regarding stage migration, the LNR-based system did not show superiority to LNs-based classification.

Conclusions: Current TNM staging classification could be improved by considering the count of metastatic nodes and LNR instead of a simple record of lymph node status (N1 or N0) for colon NETs.

Keywords: Colon; neuroendocrine tumor (NET); lymph node status; prognosis

Submitted Sep 11, 2020. Accepted for publication Nov 25, 2020.

doi: 10.21037/jgo-20-444

View this article at: http://dx.doi.org/10.21037/jgo-20-444 


\section{Introduction}

Colon neuroendocrine tumors (C-NETs) are a series of rare and highly heterogeneous neoplasms, accounting for approximately $0.3 \%$ of all colon malignancies (1). Neuroendocrine tumors (NETs) are derived from the diffuse neuroendocrine cell system which is made up of cells that release hormones in response to signals from the nervous system. The clinical course of these diseases varies widely from asymptomatically indolent lesions to fatally aggressive ones with carcinoid syndrome. Most patients are small in size and have no obvious symptoms. If the tumor grows to a certain size or grows in a special location, it can often cause intestinal dysfunction, abdominal pain or obstruction symptoms of different degrees. However, these symptoms were not significantly different from those caused by colorectal adenocarcinoma in the same location.

Therefore, a precise staging system is pivotal for perfecting outcomes by more accurately stratifying, hence more appropriately and correctly delivering treatment to highrisk patients to prevent recurrence or mortality. However, the current widely-used TNM staging system proposed by two societies, the European Neuroendocrine Tumor Society (ENETS) and the American Joint Committee on Cancer (AJCC), roughly classifies C-NETs into two groups, N1 or N0. The classification is with the presence or absence of regional lymph node ( $\mathrm{LN}$ ) involvement, instead of giving precise $\mathrm{N}$ staging information $(2,3)$. Obviously, prognostic heterogeneity should not be neglected among individual patients (e.g., N1 stage); yet, further precise stratification of patients in this group is warranted. Recent studies on many other NETs and solid tumors, including gastrointestinal and breast cancers, have unequivocally validated the importance of the number of positive lymph nodes (LNs) and the ratio between positive nodes and removed nodes (LNR) in determining patient outcomes (4-8). However, it remains unclear whether these two factors provide additional prognostic information for patients with C-NETs owing to the low intrinsic incidence of this disease. In this study, we hypothesized that higher LNs and LNR levels are associated with worse prognosis, and we used the National Cancer Institute's Surveillance, Epidemiology, and End Results (SEER) database to investigate the predictive power of these two parameters among patients with C-NETs.

We present the following article in accordance with the STROBE reporting checklist (available at http://dx.doi. org/10.21037/jgo-20-444).

\section{Methods}

\section{Cobort selection strategy}

The study cohort was found from the SEER registry of the National Cancer Institute. Our cohort was restricted to patients with histologically confirmed NETs of the colon (C18.0-C18.9, excluding the appendix), corresponding SEER ICD-O-3 histology codes 8150 to 8156,8240 to 8250 , and $9091(4,9,10)$. To eliminate most of the pediatric tumors, we omitted patients aged 18 and younger from the analysis. We also excluded patients with prior history of malignancies; those are receiving neoadjuvant radiotherapy or those with unknown neoadjuvant radiotherapy status; cases from nursing homes, autopsies, and death certificates; those with distant metastasis; and cases that lack detailed information on regional lymph nodes, including the number of LNs harvested and the number of LNs involved. We further limited our analysis to surgically resected (segmental, hemicolectomy, or total colectomy) cases and excluded those who had no cancerdirected surgery or those with no information on whether cancer-directed surgery performed, and those who had local excision. To identify the surgery procedure, we reviewed the SEER data using the SEER "site-specific surgery" variables for 1988 to 1997 (surgery codes 30-70) and "surgery of primary site" variables for 1998 to 2011 (surgery codes $30-80$ ). Please refer to Table S1 for more details. Finally, 1,242 patients were included, forming 391 stage N0 patients and 851 stage N1 patients. Patients with N0 constituted the reference group. We also found stage IV patients as another reference group. After excluding the patients with primary lesions located in the appendix, those younger than 18 years of age, and those with a previous history of other malignancies, we had another control group with 1,032 patients. Individual stages were assigned for each patient after combining $\mathrm{T}, \mathrm{N}$, and $\mathrm{M}$ categories with ENETS/AJCC proposed TNM staging classification. Colon cancers are further divided into the proximal colon (cecum, ascending colon, hepatic flexure, and splenic flexure) and distal colon (descending and sigmoid colon) (11). The patient selection flow chart is shown in Figure 1. The institutional ethics review board approved this study of HwaMei Hospital (Ningbo No. 2 Hospital), Ningbo, China. The study was conducted in accordance with the Declaration of Helsinki (as revised in 2013). 


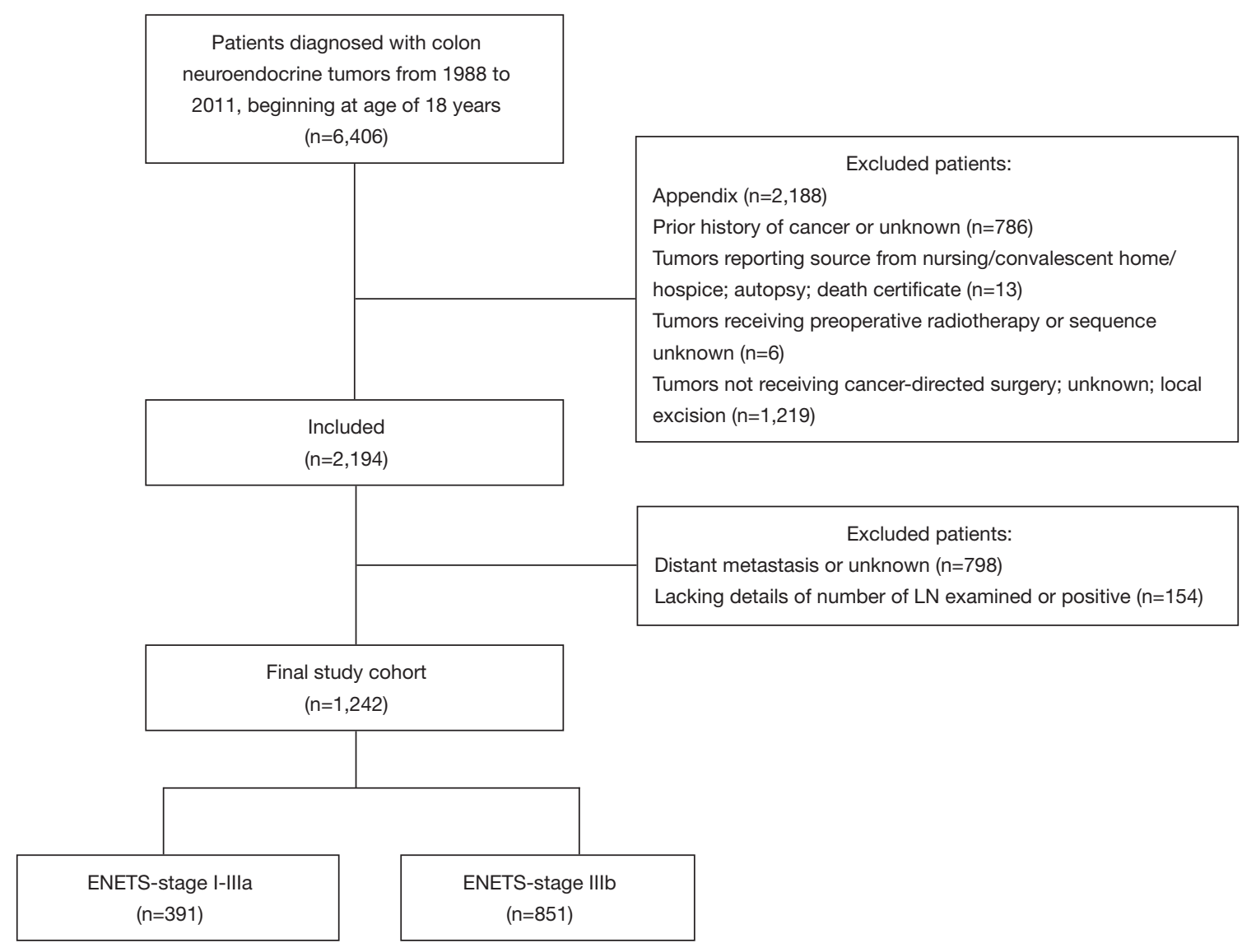

Figure 1 Patient selection flow diagram.

\section{Statistical analysis}

The distribution of continuous variables is reported as the mean value and standard deviation. Categorical data are presented as numbers and proportions. The optimal thresholds of LNs and LNR were calculated by Harrell's C statistic (C-index) $(12,13)$, and we suggested $\leq 4,>4-10,>10-13$, and $>13$ for LNs-based classification (Table S2); and $\leq 0.51,>0.51-0.71$, and $>0.71$ for LNR-based categories (Table S3). Since we could not find the optimal cutoff number for LNs harvested, we categorized it into three groups as $\leq 10,>10-15,>15$, according to a previous study (6). Comparisons among subgroups of continuous data were tested with the Kruskal-Wallis rank-sum test for any non-normal distribution. Chi-square test or Fisher's exact tests were used for categorical data comparisons, as necessary. The cancer-specific survival (CSS) was considered the primary endpoint of this study. Kaplan-Meier method and log-ranking tests were used to evaluate the difference in survival time among curves, and after excluding missing data on demographics and pathology results, a stepwise Cox proportional hazard regression was performed for multivariate survival analysis. All tests were two-sided and statistical significance was set at $\mathrm{P}<0.05$. All the statistical analyses were performed using R software ver. 3.1.3 (http:// www.R-project.org) with libraries for survival, rms, dynpre, and compare C and SPSS version 17.0 for Windows (SPSS Inc. Chicago, IL, USA).

\section{Results}

\section{Demographic and clinicopathological characteristics}

A total of 851 patients were included in the analysis, consisting of $382(45 \%)$ male and 469 (55\%) female patients, with a median age of 64 years (range, 21-94 years). Eighty-nine percent of the tumors were in the proximal colon, and $11 \%$ of the tumors were found in the distal colon. The median number of metastatic lymph nodes and the number of lymph nodes removed was 3 (range, 
1-37) and 12 (range, 3-63). The median LNR was 0.33 (range, $0.02-1$ ) in our study. The median follow-up was 23 months, ranging from 0 to 279 months. Of the 851 patients, $542(64 \%), 252(30 \%), 24(3 \%)$, and $33(33 \%)$ had $\leq 4,>4-10,>10-13$, and $>13$ LNs, respectively. 626 (74\%), $89(10 \%)$ and $136(16 \%)$ of the cohort had an LNR of $\leq 0.51,>0.51-0.71$, and $>0.71$, respectively. Table 1 summarizes the distribution of clinicopathological data according to LNs and LNR classifications. Patients who had the highest LNs and LNR also had a higher T stage (T3-4) $(\mathrm{P}<0.001)$. The patient group who had the highest LNs were more likely to have colectomy and hemicolectomy, or greater resection than partial colectomy $(\mathrm{P}=0.012)$. Regarding the LNR category, most patients with the highest level had hemicolectomy or greater resection than partial colectomy or colectomy $(\mathrm{P}=0.005)$. Additionally, patients in the highest LNs group had a propensity to present with a distal colon $(\mathrm{P}<0.001)$. Distribution was comparable from age $(\mathrm{P}=0.084$, LNR; $\mathrm{P}=0.576$, LNs), gender $(\mathrm{P}=0.647$, LNR; $\mathrm{P}=0.920, \mathrm{LNs})$, race/ethnicity $(\mathrm{P}=0.092$, LNR; $\mathrm{P}=0.254$, LNs $)$ and marital status $(\mathrm{P}=0.142$, LNR; $\mathrm{P}=0.746$, LNs) for either LNs or LNR subgroup.

\section{Survival analysis}

Figure 2 shows the observed 10-year NETs-specific survival rates of all the patients stratified by LNs classification. The cancer-specific survival rate decreased when the number of LNs increased. The 10 -year survival rates were $78.3 \%(95 \%$ CI, $74.2-82.6 \%$ ), 61.3\% (95\% CI, $52.4-71.7 \%$ ), $40.8 \%$ (95\% CI, 20.7-80.7\%) for patients in the $\leq 4,>4-10$, and $>10-13$ LNs groups, respectively. For patients in a $>13 \mathrm{LNs}$ group, 10-year survival rates were not available because the maximum follow-up in this group was 108 months, and it appeared none of the patients survived more than ten years. When patients were staged according to LNR, a similar significant trend in survival rates was observed. The observed 10-year survival rates were $79.9 \%$ (95\% CI, 74.8-85.5\%) for LNR, $\leq 0.51,57.4 \%$ (95\% CI, 43.8-75.2\%) for LNR, $>0.51-0.71$, and $40.0 \%$ (95\% CI, 31.0-51.5\%) for LNR, $>0.71$. As a comparison, the 10 -year survival rates for patients with $\mathrm{N} 0$ and distant metastasis were $80.0 \%$ (95\% CI, $74.3-85.4 \%$ ) and $19.5 \%$ (95\% CI, 16.5-23.1\%), respectively, where the log-ranking comparison survival test failed to demonstrate any significant survival benefit for patients with $\mathrm{N} 0$ compared with both lowest LNs and LNR groups ( $\mathrm{P}=0.194, \mathrm{LNs} ; \mathrm{P}=0.329$, LNR). Similarly, the comparison also did not demonstrate any substantial survival benefit in the patient group with the highest LNs when compared with the distant metastasis group $(\mathrm{P}=0.547)$.

Further analysis stratified by the $\mathrm{T}$ stage, defined according to ENETS/AJCC classification, demonstrated that the prognosis significantly worsened with an increase in LNs and LNR among patients with T3-T4 $(\mathrm{P}<0.001$ for both), but not among those with T1-T2 ( $\mathrm{P}=0.629$, LNs; $\mathrm{P}=0.143$, LNR) (Figure 3). To be more specific, among those with T3-T4 disease, patients with $\mathrm{LNR} \leq 0.51$ had a 10 -year survival of $75.6 \%$ (95\% CI, 71.3-80.4\%) compared to $57.1 \%$ (95\% CI, $43.3-75.3 \%$ ) for $\mathrm{LNR}>0.51-0.71$ and $37.2 \%$ (95\% CI, $28.3-49.0 \%$ ) for $\operatorname{LNR}>0.71$. The 10 -year survival rate was $74.1 \%$ (95\% CI, 69.2-79.4\%) among patients with $\mathrm{LNs} \leq 4$, and it dramatically decreased to $57.2 \%$ (95\% CI, $47.5-68.7 \%)$ and $40.8 \%$ (95\% CI, $20.7-80.7 \%$ ) among patients with $\mathrm{LNs}>4-10$ and $>10-13$. Further, multiple analyses also revealed an added impact that LNs and LNR status has on NETs-related survival from $\mathrm{T}$ status. In patients with T3-T4 status, the hazard ratio of fatalities was 1.25 (95\% CI, 0.99-1.79), 1.66(95\% CI, 0.77-3.57), and 3.47 (95\% CI, 2.00-6.02) for those with LNs $>4-10,>10-13,>13$, and 1.23 (95\% CI, 0.75-2.00), 2.07 (95\% CI, 1.40-3.05) for those with LNR, $>0.51-0.71$, and $>0.71$, respectively (Table 2). However, LNs and LNR did not prove a significant effect on cancer-specific survival rates among patients with T1-2 status.

We surmised it was essential to evaluate the possible effect of the number of LNs removed or harvested on the survival rate. However, whereas to the earlier studies, we failed to demonstrate any positive effect (i.e., the greater the number of LNs resected, the better the prognosis). Therefore, an LNR-based system does not show higher prognostic validity than an LNs-based classification (Figure 4).

\section{Discussion}

Currently, the presence of lymph node involvement has been demonstrated as a poor predictor for outcomes of C-NETs, and the two widely accepted tumor-nodemetastasis staging systems uniformly classify patients with lymph node involvement into the stage IIIB group. However, advanced stratification of metastatic lymph nodes is indispensable for correct staging and prognostic estimation in clinical practice.

There is rapidly increasing evidence the number of positive LNs and LNR is an important determinant of prognosis in gastroenterological cancer. The current 7 th 


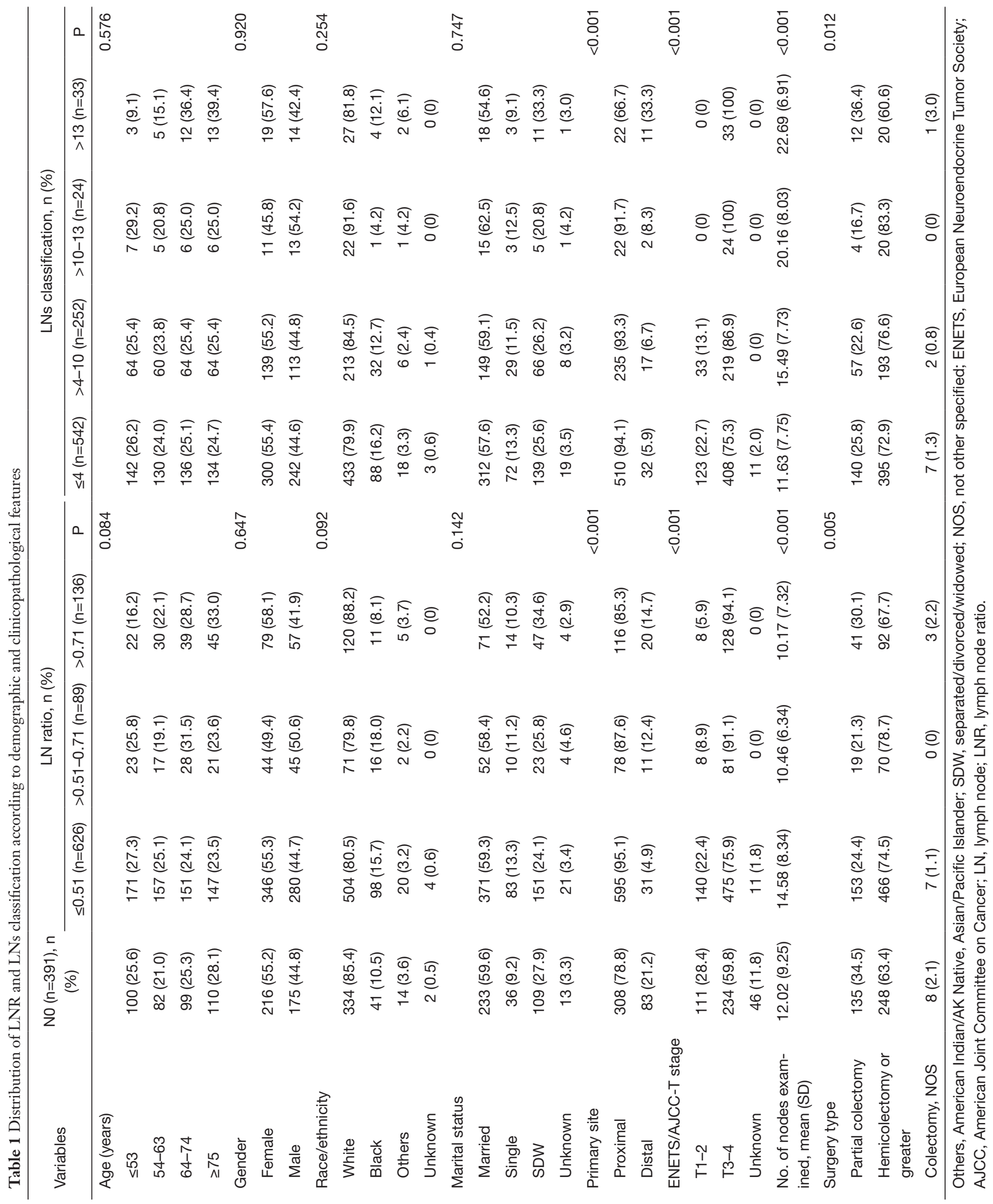



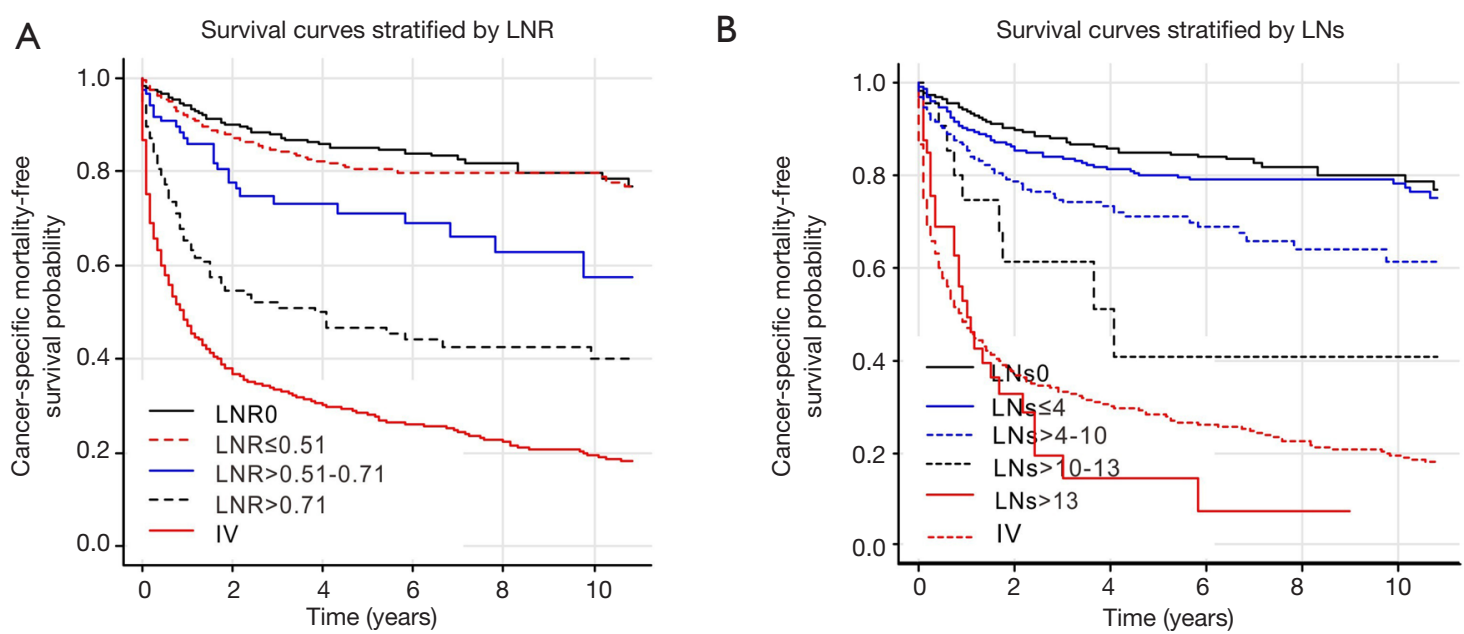

Figure 2 Cancer-specific survival curves stratified by LNR (A) and LNs (B). Patients with node-negative and distant metastasis as the reference group. LNR, lymph node ratio; LN, lymph node.

edition of the AJCC staging system uses the number of positive lymph nodes further to divide the pN stage into many solid cancers. However, there has been no earlier report addressing the relationship between the extent of lymph node involvement and prognosis in C-NETs. To our knowledge, this is believed to be the first and largest retrospective study showing a prognostic significance of LNs and LNR for colon NETs patients.

In this study, we demonstrated the most appropriate cutoff points of LNs for prognosis as 4, 10, and 13 . We found the prognosis of patients with $\mathrm{LNs}$ value $\leq 4$ paralleled the prognosis of patients with node-negative and is significantly better than the prognosis of patients with LNs $>4-10,>10-13$, and distant metastasis.

$\mathrm{LNR}$, as a reproducible and reliable prognostic indicator, was first described in gastric cancer studies $(7,14-17)$. Recent evidence also implicated the LNR as a prominent prognostic factor, and it should be taken into consideration in the future for stratification schemes. Bando et al. $(15,16)$ also demonstrated LNR-based classification, presented a superior capability of precisely staging and predicting survival compared with both the AJCC and JGCA proposed $\mathrm{pN}$-based categories. The prognostic value of LNR in the colon was first proved by Berger et al. (6), who analyzed 3,759 stage II-III colon adenocarcinomas and stratified patients into four groups on quartiles ( $L N R \leq 0.05$, $0.05-0.19,0.2-0.39$, and $>0.4)$. The 5 -year cancer-specific survival rates were $79 \%, 73 \%, 63 \%$, and $52 \%$, respectively. Concerning NETs, Kim et al. (4) analyzed 2,984 patients with small intestinal NETs at the AJCC/ENETS IIIB stage and reported 10-year NET-specific survival as $85 \%, 77 \%$, and $74 \%$ for patients in the $\leq 0.2,>0.2-0.5$, and $>0.5$ LNR groups, respectively. Similarly, Tang et al. (5) analyzed 79 gastric neuroendocrine carcinoma patients who underwent D2 lymphadenectomy and reported that LNR was an independent prognostic factor, revealing significantly different prognoses between $\leq 10 \%$ and $>10 \%$ of LNR. The limitation of using LNR was insufficient specific cutoffs reached by consensus, and the method used to find this threshold varied widely. In our study, a specific statistical method was used to determine the optimal cutoff; a series of log-ranking tests were performed, using successive LNR cutoffs (0.03 to 0.99), and the predictive efficiency of each cutoff was evaluated by the Harrell's C statistic, which resulted in cutoff values of 0.51 and 0.71 .

There have been two controversial views regarding the prognostic role of lymphadenectomy: (I) whether lymphadenectomy is only a tool for achieving more accuracy in staging with no therapeutic value, and (II) whether it is beneficial for prognosis (18). When many LNs were removed, it not only resulted in an incomplete excision of the tumor and draining area but also allowed for a thorough examination of the specimen and more accurate staging, which resulted in the prevention of stage migration, known as the Will Rogers phenomenon $(19,20)$. There is consensus on the retrieval of at least 12 lymph nodes in colon adenocarcinoma. LNR, a single parameter combining both the number of lymph nodes evaluated and the positive nodes, is superior to the AJCC classification, specifically for those patients with inadequate LN resected 

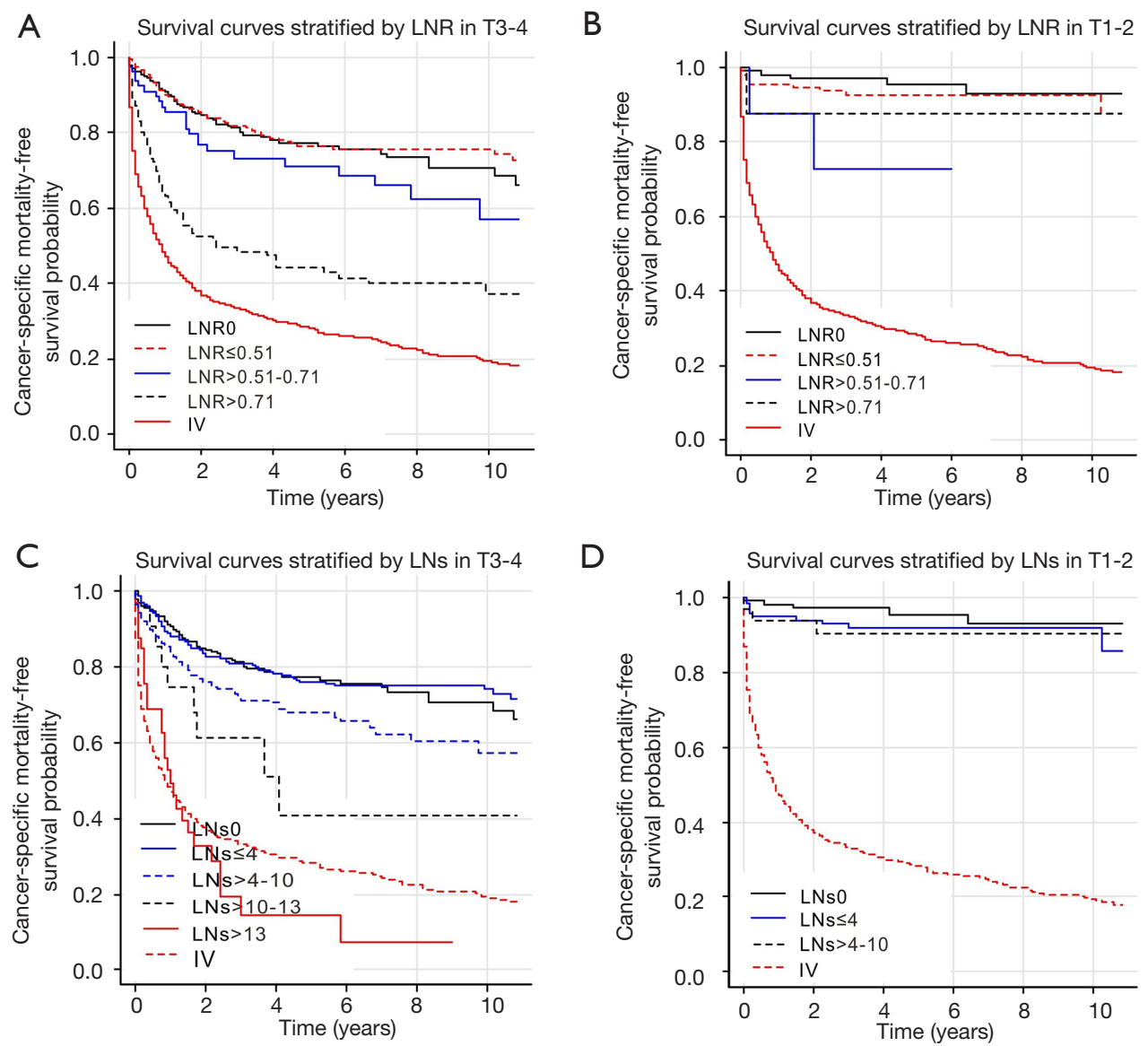

Figure 3 Cancer-specific survival curves in patients of different LNR and LNs categories after being stratified by the ENETS/AJCC T stage. (A) Survival curves stratified by LNR in patients with T3-4 disease; (B) survival curves stratified by LNR in patients with T1-2 disease; (C) survival curves stratified by LNs in patients with T3-4 disease; (D) survival curves stratified by LNs in patients with T1-2 disease. LN, lymph node; LNR, lymph node ratio; ENETS, European Neuroendocrine Tumor Society; AJCC, American Joint Committee on Cancer.

Table 2 Multiple analysis stratified by T status

\begin{tabular}{|c|c|c|c|c|}
\hline Covariate & \multicolumn{2}{|c|}{ T1-2 } & \multicolumn{2}{|c|}{ T3-4 } \\
\hline LNs category (ref. $\leq 4$ ) & - & - & & \\
\hline$>4-10$ & - & - & $1.25(0.99-1.79)$ & 0.216 \\
\hline$>10-13$ & - & - & $1.66(0.77-3.57)$ & 0.190 \\
\hline \multicolumn{5}{|c|}{ LNR category (ref. $\leq 0.51$ ) } \\
\hline$>0.51-0.71$ & - & - & $1.23(0.75-2.00)$ & 0.405 \\
\hline$>0.71$ & - & - & $2.07(1.40-3.05)$ & $<0.001$ \\
\hline
\end{tabular}

Adjustment of age, gender, race, marital status, T stage and No. of LN removed. LN, lymph node; LNR, lymph node ratio. 


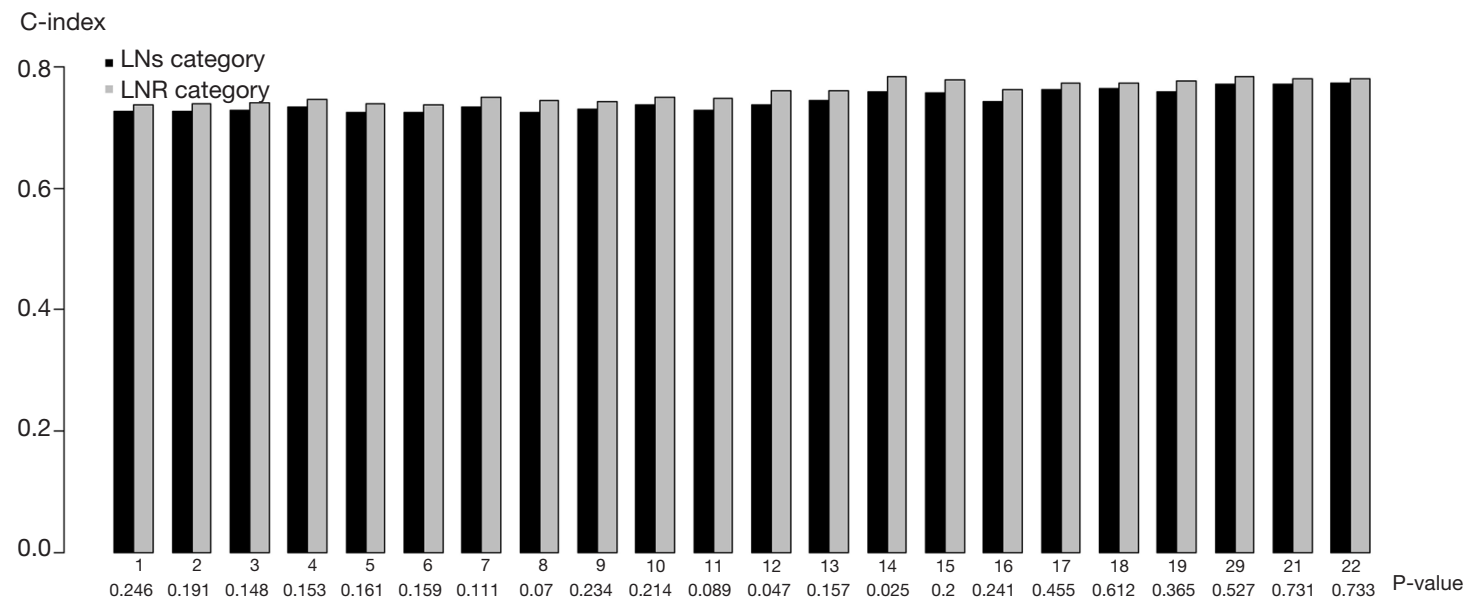

Number of nodes removed

Figure 4 Comparing the predictive capacity of LNR and LNs for all patients after considering the successively minimal number of LNs removed. LN, lymph node; LNR, lymph node ratio.

$(7,15,16)$. In our study, we did not ascertain the number of LNs removed as a significant variable affecting survival. Although the predictive ability of LNR categories was always higher than the LNs categories, the $\mathrm{p}$ values between them were insignificant regardless of the minimal number of LNs removed. The LNR-based system also did not show superiority to LNs-based classification even when we limited our analysis to patients with T3-4 (Figure S1).

There are some intrinsic limitations of using the SEER database. First, the database lacks information on preoperative chemotherapy that affects the amount of lymph node metastasis, thereby influencing the lymph nodes removed. Secondly, information on tumor proliferative ability evaluated by either Ki-67 index or mitotic count was unavailable in the database. The goodness of fit for the Cox model and the value of the $\mathrm{C}$-index might be increased after considering these two parameters during multiple analyses. However, these indices were not assessed until 2010 (21), and most NETs located in the colon present a low-level of mitotic count and Ki-67 labeling index, suggesting that these two factors have a limited confounding effect on the entire model. Additionally, external validation using other cohorts is needed.

In general, our findings suggest the LNs-based and LNR-based classifications are significantly critical prognostic factors for NETs in the colon and serve as indispensable tools for further differentiation of the nodepositive patients.

\section{Acknowledgments}

Funding: This work was supported by the Key Laboratory of Diagnosis, Treatment, and Research of Digestive System Tumor of Zhejiang Province (2019E10020), the Natural Public Welfare Fund of Zhejiang Province (LGC20H160002), the Medical and Health Science and Technology Foundation of Zhejiang Province (2019KY595, 2018KY690, 2018KY699, 2017KY593, 2017KY594), the Natural Science Foundation of Ningbo (2018A610368, 2017A610145, 2017A610158, 2016A610135).

\section{Footnote}

Reporting Checklist: The authors have completed the STROBE reporting checklist. Available at http://dx.doi. org/10.21037/jgo-20-444

Conflicts of Interest: All authors have completed the ICMJE uniform disclosure form (available at http://dx.doi. org/10.21037/jgo-20-444). The authors have no conflicts of interest to declare.

Ethical Statement: The authors are accountable for all aspects of the work in ensuring that questions related to the accuracy or integrity of any part of the work are appropriately investigated and resolved. The institutional ethics review board approved this study of HwaMei Hospital (Ningbo No. 2 Hospital), Ningbo, China. The study was conducted in accordance with the Declaration of 
Helsinki (as revised in 2013). The written informed consent was waived due to the retrospective nature of the study.

Open Access Statement: This is an Open Access article distributed in accordance with the Creative Commons Attribution-NonCommercial-NoDerivs 4.0 International License (CC BY-NC-ND 4.0), which permits the noncommercial replication and distribution of the article with the strict proviso that no changes or edits are made and the original work is properly cited (including links to both the formal publication through the relevant DOI and the license). See: https://creativecommons.org/licenses/by-nc-nd/4.0/.

\section{References}

1. Bernick PE, Klimstra DS, Shia J, et al. Neuroendocrine carcinomas of the colon and rectum. Dis Colon Rectum 2004;47:163-9.

2. Landry CS, Brock G, Scoggins CR, et al. Proposed staging system for colon carcinoid tumors based on an analysis of 2,459 patients. J Am Coll Surg 2008;207:874-81.

3. Rindi G, Kloppel G, Couvelard A, et al. TNM staging of midgut and hindgut (neuro) endocrine tumors: a consensus proposal including a grading system. Virchows Arch 2007;451:757-62.

4. Kim MK, Warner RR, Ward SC, et al. Prognostic significance of lymph node metastases in small intestinal neuroendocrine tumors. Neuroendocrinology 2015;101:58-65.

5. Tang X, Chen Y, Guo L, et al. Prognostic significance of metastatic lymph node number, ratio and station in gastric neuroendocrine carcinoma. J Gastrointest Surg 2015;19:234-41.

6. Shah CP, Mramba LK, Bishnoi R, et al. Survival trends of metastatic small intestinal neuroendocrine tumor: a population-based analysis of SEER database. J Gastrointest Oncol 2019;10:869-77.

7. Liu DJ, Hua R, Sun YW. Current treatment status in pancreatic neuroendocrine neoplasms. Chin Clin Oncol 2019;8:20.

8. Weir L, Speers C, D'Yachkova Y, et al. Prognostic significance of the number of axillary lymph nodes removed in patients with node-negative breast cancer. J Clin Oncol 2002;20:1793-9.

9. Yao JC, Hassan M, Phan A, et al. One hundred years after "carcinoid": epidemiology of and prognostic factors for neuroendocrine tumors in 35,825 cases in the United States. J Clin Oncol 2008;26:3063-72.

10. Kim MK, Warner RR, Roayaie S, et al. Revised staging classification improves outcome prediction for small intestinal neuroendocrine tumors. J Clin Oncol 2013;31:3776-81.

11. Manne U, Myers RB, Moron C, et al. Prognostic significance of Bcl-2 expression and $\mathrm{p} 53$ nuclear accumulation in colorectal adenocarcinoma. Int J Cancer 1997;74:346-58.

12. Harrell FE, Lee KL, Mark DB. Multivariable prognostic models: issues in developing models, evaluating assumptions and adequacy, and measuring and reducing errors. Stat Med 1996;15:361-87.

13. Downing SR, Cadogan KA, Ortega G, et al. The number of lymph nodes examined debate in colon cancer: how much is enough? J Surg Res 2010;163:264-9.

14. Celen O, Yildirim E, Berberoglu U. Prognostic impact of positive lymph node ratio in gastric carcinoma. J Surg Oncol 2007;96:95-101.

15. Bando E, Yonemura Y, Taniguchi K, et al. Outcome of ratio of lymph node metastasis in gastric carcinoma. Ann Surg Oncol 2002;9:775-84.

16. Inoue $\mathrm{K}$, Nakane $\mathrm{Y}$, Iiyama $\mathrm{H}$, et al. The superiority of ratio-based lymph node staging in gastric carcinoma. Ann Surg Oncol 2002;9:27-34.

17. Du D, Han Z, Lian D, et al. The value of preoperative lymphocytes-to-monocytes ratio in predicting lymph node metastasis in gastric cancer. Transl Cancer Res 2019;8:2053-8.

18. Sigurdson ER. Lymph node dissection: is it diagnostic or therapeutic? J Clin Oncol 2003;21:965-7.

19. Le Voyer TE, Sigurdson ER, Hanlon AL, et al. Colon cancer survival is associated with increasing number of lymph nodes analyzed: a secondary survey of intergroup trial INT-0089. J Clin Oncol 2003;21:2912-9.

20. Feinstein AR, Sosin DM, Wells CK. The Will Rogers phenomenon. Stage migration and new diagnostic techniques as a source of misleading statistics for survival in cancer. N Engl J Med 1985;312:1604-8.

21. Moon CM, Huh KC, Jung SA, et al. Long-Term Clinical Outcomes of Rectal Neuroendocrine Tumors According to the Pathologic Status After Initial Endoscopic Resection: A KASID Multicenter Study. Am J Gastroenterol 2016;111:1276-85.

(English Language Editor: J. Chapnick)

Cite this article as: Xiao C, Song B, Yi P, Xie Y, Li B, Lian P, Ding S, Lu Y. Deaths of colon neuroendocrine tumors are associated with increasing metastatic lymph nodes and lymph node ratio. J Gastrointest Oncol 2020;11(6):1146-1154. doi: $10.21037 /$ jgo-20-444 


\section{Supplementary}

Table S1 Corresponding SEER code of surgery

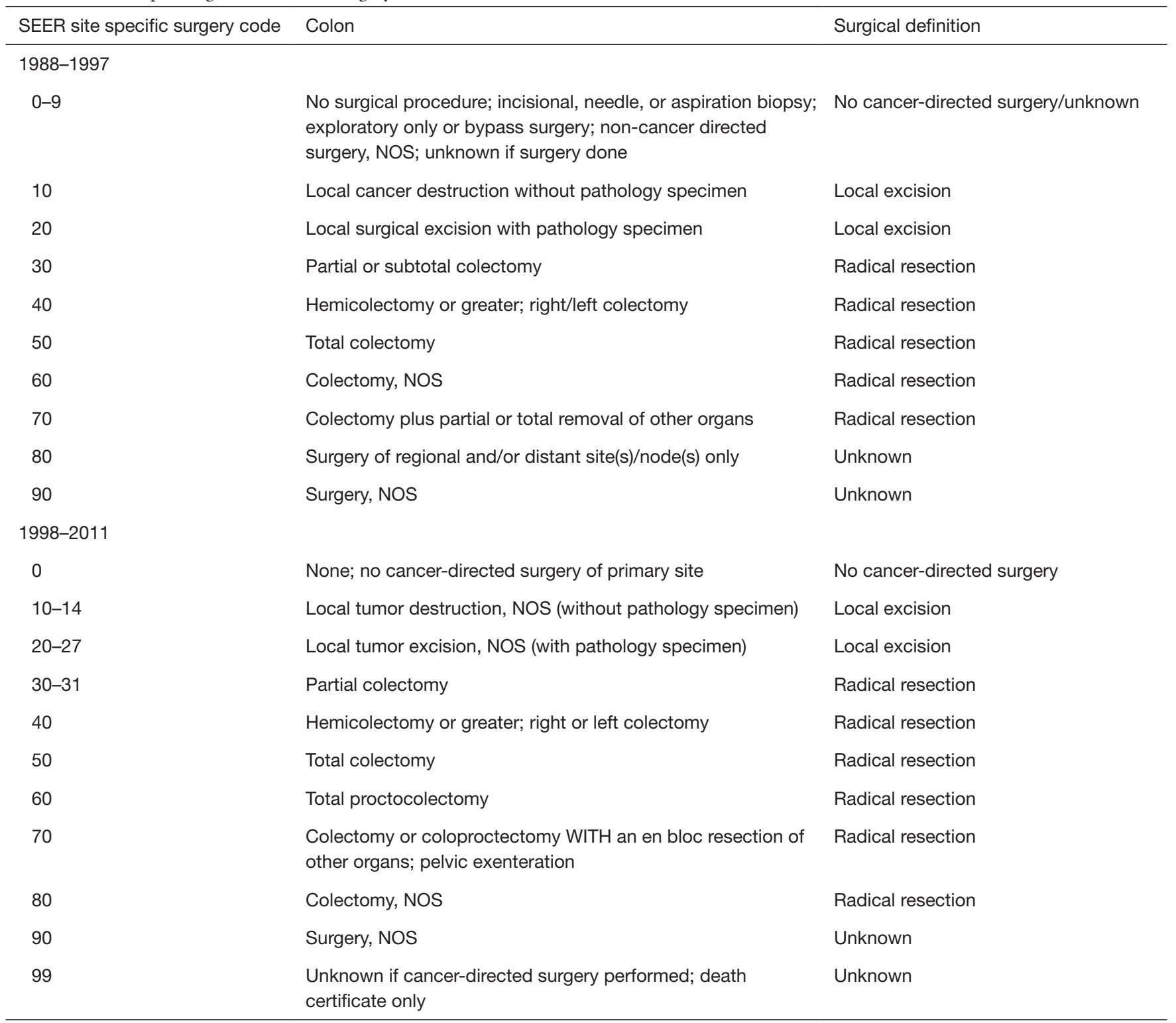

(C) Journal of Gastrointestinal Oncology. All rights reserved. 
Table S2 Calculation the cutoff of LNs

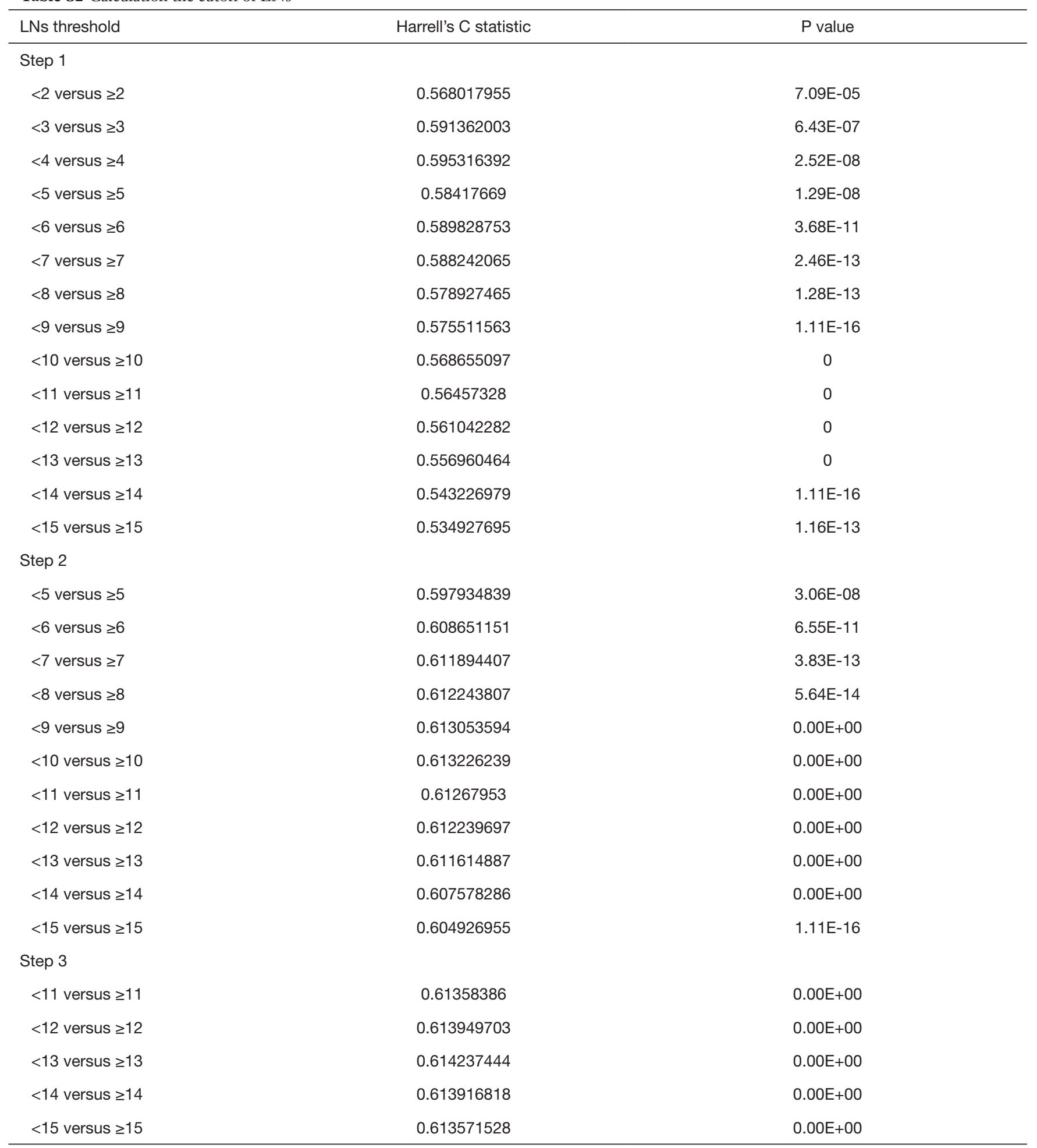

(C) Journal of Gastrointestinal Oncology. All rights reserved. 


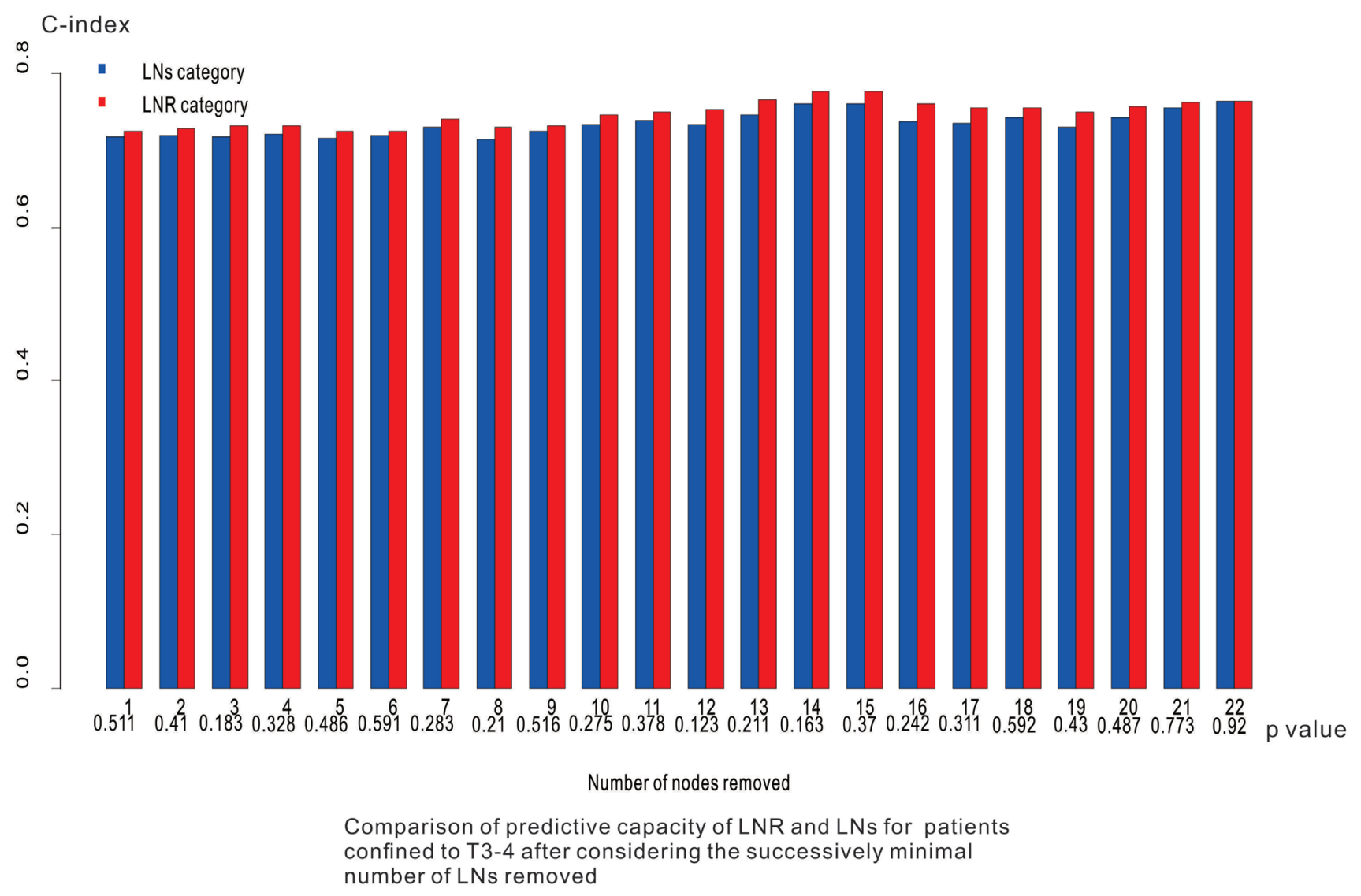

Figure S1 Comparison of predictive capacity of LNR and LNs for patients confined to T3-4. LN, lymph node; LNR, lymph node ratio. 